\title{
$O$ debate como gênero textual a ser fomentado nas aulas de línguas
}

\author{
Vera Lúcia Lopes CRISTÓVÃO \\ Adja Balbino de Amorim Barbieri DURÃO \\ Elvira Lopes NASCIMENTO \\ Universidade Estadual de Londrina
}

Palavras chave: gênero textual; debate; interaccionismo sócio-discursivo.

Resumo: No presente trabalho, propõe-se a inclusão do "Debate" como gênero textual a ser potencializado nos processos de ensino/aprendizagem de língua materna e línguas estrangeiras, face às necessidades comunicativas a serem desenvolvidas ou aperfeiçoadas no contexto escolar brasileiro na perspectiva do interacionismo sócio-discursivo.

\begin{abstract}
The present work proposes the inclusion of "Debates" as a text genre to be developed in the processes of teaching-learning a mother tongue and foreign languages due to the communicative needs to be acquired or improved in the brazilian school context within the socio-discoursive interactional perspective.

Resumen: En el presente estudio, se propone la inclusión del "Debate" un como género textual que debe ser fomentado en los procesos de enseñanza/ aprendizaje de lengua materna y lenguas extranjeras, teniendo en cuenta las necesidades comunicativas que deben desarrollarse o perfeccionarse en el contexto escolar brasileño según la perspectiva del intercaccionismo sociodiscursivo.
\end{abstract}

SIGNUM: Estud. Ling., Londrina, n. 5, p. 125-157, dez. 2002 125 


\section{Introdução}

O conceito de gêneros discursivos, advindo de Bakhtin (1929/79; 1979/92), tem como ponto de partida a obra Estética da criação verbal, apesar de já aparecer esboçado em Marxismo e filosofia da linguagem. Da proposta inicial de Bakhtin, na qual a enunciação é vista como produto de interação social, quer se trate de um ato de fala determinado pela situação imediata ou pelo contexto que constitui o conjunto das condições de vida de uma determinada comunidade lingüística, às visões mais recentes e revistas sobre os gêneros discursivos (do discurso, textuais ou de texto) permaneceu uma constante: a comprovação de uma dependência indissociável das mensagens produzidas em uma língua específica por um emissor (que resulta de suas condições de produção) ao destinatário (que depende de suas condições de recepção), ao momento histórico e ao contexto sóciocultural no qual essa mensagem se forja (que envolve, entre outras coisas, as condições de circulação).

A idéia de gêneros discursivos (doravante gêneros textuais) é magistralmente abordada por Bronckart (1999), que diz que a linguagem é um elemento da atividade social humana cuja função primeira é de ordem comunicativa ou pragmática, de modo que as representações verbais são "o produto de uma reformulação coletiva imposta às representações até então idiossincráticas”. Assim sendo, a atividade lingüística se organiza em discursos ou textos e esses, diversificam-se em gêneros.

A discussão sobre gêneros textuais é retomada nos Parâmetros Curriculares Nacionais para o Ensino Fundamental de Língua Portuguesa PCN's (p. 7), sendo colocada como base de uma proposta que leva em conta as necessidades comunicativas a serem desenvolvidas ou aperfeiçoadas no contexto escolar brasileiro:

Produzir linguagem significa produzir discursos: dizer alguma coisa a alguém, de uma determinada forma, em um determinado contexto 
histórico e em determinadas circunstâncias de interlocução. Isso significa que as escolhas feitas ao produzir um discurso não são aleatórias - ainda que possam ser inconscientes-, mas decorrentes das condições em que o discurso é realizado. [...] isso tudo determina as escolhas do gênero no qual o discurso se realizará, dos procedimentos de estruturação e da seleção dos recursos lingüísticos.

Portanto, diga o que disser, o produtor de texto (oral ou escrito) o fará no formato de um determinado gênero.

Bakhtin leva-nos a entender os gêneros textuais como resposta às formas de organização das práticas enunciativas que concretizam eventos que fazem emergir uma série de aspectos constantes no uso. Esses eventos enunciativos deixam entrever, a um mesmo tempo, o lado individual e o social do discurso, revelando, além do que já se mencionou, a mobilização de estruturas comunicativas e semióticas comuns aos textos afiliados a um certo gênero, o domínio de diferentes níveis de operações lingüísticas para a configuração específica desse gênero, assim como a utilização de conjuntos de seqüências textuais e tipos discursivos que constituem sua estrutura.

A questão do emprego dos gêneros textuais como emolduramento para os eventos enunciativos leva a uma constatação curiosa: qualquer enunciado que se proponha não é totalmente original no sentido de que repete o gênero usado em contextos similares aos dessa enunciação. Essa "falta de originalidade", entretanto, é apenas aparente, já que todos os produtores de texto movem-se com a finalidade de ajustar os textos que propõem aos formatos socialmente revalidados, considerando suas próprias necessidades expressivas, assim como as de seu destinatário, situando-se a si e a seu destinatário em um contexto determinado, ou como assinala Fäita (1997, p. 165): "cada texto é individual na medida em que pode-se impor um estilo pessoal que represente a relação do enunciador com a que utiliza." 
Uma das metas dos professores de línguas deve ser, segundo nosso ponto de vista, trabalhar, em sala de aula, com a maior quantidade possível de gêneros textuais, visto que pretende-se que o alunado a expanda sua performance para elaborar e interpretar textos em sua língua materna e em outras línguas que estude, portanto, os gêneros, além de serem instrumentos de comunicação, são, nos contextos de ensino, objetos de ensino/aprendizagem (Schneuwly; Dolz, 1997).

Bakhtin (apud Fäita, 1997) chama a nossa atenção para a impossibilidade de trabalhar com todos os gêneros textuais existentes, posto que são "tão variados como as próprias esferas da atividade humana", portanto, à tarefa de fomento dos gêneros textuais na sala de aula, precede a de seleção, seleção essa que coloca em alça de mira os textos autênticos, ou seja, enunciados propostos em contextos reais de comunicação.

No presente trabalho, propomos a inclusão do "Debate" como gênero textual a ser potencializado nos processos de ensino e aprendizagem tanto de língua materna como de língua estrangeira. A justificativa dessa inclusão é a possibilidade de desenvolver a capacidade argumentativa dos alunos, ajudando-os a estar aptos a se posicionar sobre os mais variados problemas sociais que invariavelmente surgem em sua vida diária, de um modo eficaz e ajustado às demandas da sociedade na qual vivem, ou daquelas que formam os contextos das línguas estrangeiras que estudam, das quais, direta ou indiretamente, tomam parte.

\section{Caracterizando o "Debate"}

No Brasil, constantemente, deparamo-nos com debates em programas de tipo talk show ou "torneios verbais" entre oponentes veiculados pela televisão. Esse tipo de embate é caracterizado por Schneuwly e Dolz (1999) como eventos que colocam em oposição interlocutores que usam, além dos próprios mecanismos lingüísticos, meios como a "teatralização", o "o sensacionalismo", "a verbalização de insultos 
e, até, de semiverdades", além do emprego da "contradição com o fim de dominar", ou, ainda, de "ridicularizar" o adversário. A escuta do oponente, por sua vez, tem por fim identificar brechas na fala do adversário que lhe permitam desarmar seu predecessor, com o fim de "fazer triunfar, a qualquer preço, uma posição em detrimento da outra". Muito embora seja um fato que essa tipologia é a prototípica do debate como gênero textual que costumamos ver nos meios de comunicação, acreditamos ser possível desenvolver, junto aos alunos, uma forma de debate menos "belicosa" e que favoreça a ampliação de suas habilidades argumentativas, com vistas a saber "defender um ponto de vista, uma escolha ou um procedimento de descoberta" (Schneuwly; Dolz, 1999).

Debater é, num evento dialógico e polêmico, defender pontos de vista com o fim de fazer o interlocutor aderir ao que se propõe. Quando se mobilizam procedimentos com vistas a levar o interlocutor a crer no que se diz ou a fazer o que se propõe, geralmente se utilizam recursos argumentativos, alguns dos quais podem ser:

a) manter a unidade textual, já que um texto dispersivo e confuso faz com que o interlocutor tenha dificuldade para identificar sua(s) idéia(s) central(is);

b) comprovar a(s) idéia(s) defendida(s) mediante a citação de trabalho(s) de autor de referência, conferindo confiabilidade ao exposto;

c) estabelecer a relação causa-efeito entre as partes do texto mediante mecanismos de coesão textual;.

d) confirmar o que se diz mediante exemplos;

e) refutar idéias.

Aspectos pedagógicos relacionados ao desenvolvimento do gênero "Debate"

SIGNUM: Estud. Ling., Londrina, n. 5, p. 125-157, dez. 2002 
O objetivo de fomentar o debate entre estudantes com um formato produtivo do ponto de vista da argumentação é levar os alunos a encontrar soluções aceitáveis para problemas colocados em discussão através do raciocínio coletivo. As intervenções dos participantes, portanto, devem suceder-se com o fim de alcançar esclarecimentos sobre questões controversas em um processo de produção discursiva poligerada. Tal processo exige desses participantes a atenção voltada para a escuta, para a reflexão e para a assimilação dos discursos anteriores ao seu, ou seja, os debatedores devem escutar-se, discutir as tomadas de posição uns dos outros, recuperando e reformulando o já-dito por si próprios ou pelos demais, recorrendo a modalizações para dar novos ares às variadas tomadas de posição.

Como dissemos antes, além das habilidades de linguagem exigidas a propósito de qualquer tema, o debate é uma atividade que engloba capacidades discursivas, interacionais e cognitivas, já que as intervenções manifestam-se através de argumentos de vários tipos, por meio de comparações, concessões, recurso à autoridade, exemplificação, justificativa, por recorrer a diferentes formas de refutação, analogias, descrições, relatos, negociação de conflitos, formas de transmissão da palavra alheia, etc.. $\mathrm{O}$ professor, portanto, deve trabalhar para que os eventos enunciativos em sala de aula apresentem uma certa regulação interativa, o que pressupõe o respeito às regras conversacionais e à real capacidade de negociação, sem que haja prioridade dos aspectos negativos já apontados mediante a idéia prototípica dos debates freqüentemente usada nos debates televisivos.

O propósito, portanto, é que como atividade pedagógica, o debate seja desenvolvido de modo que o professor ajude os alunos a manifestar e discutir as diferentes opiniões sobre um mesmo tema, e que consigam chegar a soluções coletivas, evitando impasses ou que as diferenças terminem em conflito, ou, ao contrário, que o engajamento seja "morno" e sem motivação. Para isso, é preciso partir de descrições objetivas do gênero debate e da proposta de modelos didáticos de debate regrados, 
que definam princípios orientadores para a intervenção do professor e tornem possível uma progressão que abranja as diferentes etapas da aprendizagem desse gênero.

\section{Propriedades do gênero "Debate"}

Ao nos indagar sobre as capacidades de linguagem necessárias para a produção oral do gênero "Debate", nos baseamos na literatura já existente. Vejamos o que dizem Schneuwly e Dolz (1998) sobre os itens a serem ensinados:

Quadro 1 - Síntese do modelo didático do gênero "debate" segundo Schneuwly \& Dolz (1998)

\begin{tabular}{|c|c|c|}
\hline Capacidade de linguagem & Objetivos & Conteúdo \\
\hline Capacidade de ação & $\begin{array}{l}\text { - Familiarizar os alunos com o } \\
\text { gênero; } \\
\text { - Compreender os significados } \\
\text { da relação entre um debate e sua } \\
\text { situação de produção. }\end{array}$ & $\begin{array}{l}\text { - Definir o que é debate; } \\
\text { - Estabelecer o tema; } \\
\text { - Buscar informações sobre o } \\
\text { temas; }\end{array}$ \\
\hline Capacidade discursiva & $\begin{array}{l}\text { - Expor o aluno ao plano textual } \\
\text { global. }\end{array}$ & $\begin{array}{l}\text { - Saber diferenciar opinião de } \\
\text { argumentação; } \\
\text { - Posicionar-se a favor ou contra } \\
\text { um determinado ponto de vista. }\end{array}$ \\
\hline Capacidade lingüístico-discursiva & $\begin{array}{l}\text { - Explorar a função discursiva } \\
\text { do(s) componente(s) } \\
\text { lingüístico(s) básico(s) para o } \\
\text { gênero debate. }\end{array}$ & $\begin{array}{l}\text { - Conhecer e saber usar os } \\
\text { elementos lingüísticos adequados } \\
\text { para desenvolver-se no processo } \\
\text { do debate (marcadores } \\
\text { discursivos, funcionais, etc.) }\end{array}$ \\
\hline
\end{tabular}

Em outras palavras, os elementos a serem selecionados para o ensino e aplicados nas aulas devem:

a) explicitar a situação de comunicação e o contexto de produção do debate - capacidade de ação;

SIGNUM: Estud. Ling., Londrina, n. 5, p. 125-157, dez. 2002 
b) escolher e dispor os itens para organização interna do debate capacidades discursivas;

c) apresentar os elementos lingüísticos adequados para o desenvolvimento do debate - capacidades lingüístico-discursivas.

Quanto ao tema do gênero escolhido, deve ser interessante e permitir uma controvérsia nas opiniões. Também deve levar a uma progressão nas capacidades de linguagem dos alunos. Para isso, o trabalho didático com gêneros textuais em sala de aula prevê uma seqüência de atividades que contribuam para a progressão no desempenho dos alunos. Com esse intuito em mente, o procedimento sugerido (Schneuwly; Dolz, 1998) é de produção inicial, correções nas inadequações e produção final.

\section{A exploração do gênero "Debate" em materiais didáticos para ensino do Português, do Inglês e do Espanhol}

Os programas de ensino e materiais didáticos para o ensino de línguas são normalmente organizados por um (alguns) fator(es), a saber: ação de linguagem (compreensão e produção escrita), item gramatical (tempos verbais), campo semântico (animais), função (cumprimentar, agradecer), estratégia de leitura (skimming, scanning), gênero (debate).

O ensino baseado em gêneros textuais ainda parece ser incipiente. Com o intuito de analisar a presença ou a ausência de propostas de trabalhos didáticos com gênero para o ensino de línguas (português como língua materna, inglês e espanhol), consultamos livros didáticos e materiais suplementares comumente usados como suporte nos cursos de línguas. Como estamos realizando um trabalho conjunto de pesquisa sobre gêneros em LM e LE a serem ensinados na escola, o gênero "Debate" foi escolhido em função da nossa preocupação com o ensino da expressão oral em língua materna, entretanto a pesquisa sobre a utilização desse gênero nas aulas de LM levou-nos a investigar sua presença em materiais de LE. 


\section{a. Material para ensino da Língua Portuguesa (apêndice 1)}

Após levantamento em nove livros didáticos de Língua Portuguesa destinados a alunos de 5. a 8. ${ }^{a}$ séries do Ensino Fundamental, encontramos um único livro, de autoria de Maria Fernandes Cócco, cujo título é “ $A \not p$ novo: análise, linguagem e pensamento", que apresenta uma unidade didática que utiliza o gênero "Debate", configurada da seguinte maneira:

a) a primeira atividade trata da definição do gênero "Debate";

b) a seguir, apresentam-se três pequenos textos (em média 4 linhas)que contém "opiniões" sobre o tema de um debate virtual, já que este não é realizado depois;

c) apresentam-se duas atividades sobre a estrutura do debate (apresentador, moderador e participantes). Em relação à representação do papel do moderador, a atividade recomenda que este deve responsabilizar-se pela ordem de apresentação das idéias e deve comentar as opiniões e os argumentos apresentados. Apresenta, ainda, uma atividade que visa ao reconhecimento desses papéis;

d) propõem-se, a seguir, três atividades de escrita: 1. escrever uma opinião sobre o tema após a leitura dos pequenos textos (em média de quatro linhas); 2. escrever parágrafos que completem as expressões:" Eu acho que ..."; "Eu compreendo seu ponto de vista, mas por outro lado..." ; "Não concordo com você, pois..." e 3: escrever argumentos a favor ou contra um dado tema .

e) a última atividade proposta, solicita o planejamento de um debate em grupo sobre tema escolhido pelos alunos.

Não encontramos, nesse livro didático, qualquer referência a atividades posteriores que focalizassem as capacidades de linguagem envolvidas nesse gênero. $\mathrm{O}$ uso da linguagem argumentativa oral (instrumentos lingüísticos e discursivos que permitam aos interlocutores 
expressar/defender/refutar argumentos, os mecanismos de formulação e reformulação, incluindo aí aqueles que dizem respeito às responsabilidades enunciativas e às modalizações, a distinção entre argumento e opinião, a identificação de uma refutação, a identificação de "zonas" mais periféricas para o surgimento de conflitos, os pontos de quebra das regras conversacionais com o "roubo" do turno, a identificação de quem é a voz que "fala" em certas passagens, a força de certos argumentos, a sustentação de opiniões com um número dado de argumentos, a coerência argumentativa, a coesão nominal e verbal nas seqüências narrativas e argumentativas, a transposição do falado para o escrito, etc.) não foram enfocados em atividades que visassem ao aprofundamento e o enriquecimento de argumentos apresentados oralmente. Houve uma "fuga" em relação ao objeto de ensino: as atividades que deveriam enfocar a linguagem oral argumentativa (como preconizam os PCNs), deram lugar a atividades muito superficiais sobre o cenário do debate (a estrutura de participação), deixando de lado importantes aspectos dessa estrutura e não retomando falhas e lacunas apresentadas no comportamento lingüístico-discursivo dos alunos e que poderiam fomentar as futuras intervenções do professor.

b. Materiais para ensino da Língua Inglesa (apêndices 2, 3, 4 e 5)

Um primeiro material, de autoria de L. G. Alexander (1968/1975), tem como título "For and against". Este livro apresenta 30 ensaios cujos temas são polêmicos. Na seqüência, o autor disponibiliza argumentos e contra-argumentos com palavras-chave. Sugere um debate livre em que as idéias do livro e as próprias opiniões dos alunos sejam aproveitadas (ver apêndice 2). A presença de um mediador e o direito do público de fazer perguntas são procedimentos incentivados para uma participação proveitosa de todos. Ao final, os alunos podem votar no vencedor do debate, isto é, naqueles que desenvolveram a melhor argumentação. 
Um segundo material, de autoria de G. P. Ladousse (1983), "Speaking personally”, destinado a alunos de nível pós-intermediário, apresenta uma atividade de debate na qual se define o gênero e se ilustra uma situação que envolve um debate (ver apêndice 3). A explicação dada limita-se a estabelecer um tema e o número de pessoas que devem posicionar-se a favor ou contra uma determinada posição. Depois de um tempo de preparo de sua argumentação, aqueles que se posicionam a favor, começam, sendo seguidos por aqueles que se opõe.

Um terceiro material, de autoria de Ur (1981/1995), intitulado "Discussions that Work., discorre sobre princípios gerais que devem orientar atividades voltadas para a produção oral com fluência para, então, propor exemplos práticos, dentre eles, o debate (ver apêndice 4). Depois de levantar limitações quanto ao seu uso (os tipos de assunto e a possibilidade de participação eqüitativa dos membros de um grupo), a autora enaltece as vantagens (o gênero é apreciado por muitos além de representar uma valiosa oportunidade de aprendizagem de capacidades argumentativas) para propor alguns procedimentos.

Em primeiro lugar, Ur (1981/1995) sugere que o grande grupo seja dividido em grupos menores, cada qual com um tema que se relacione com os outros de alguma forma. Como uma primeira fase do debate, os membros de cada grupo têm um tempo pré-determinado para prepararem seus argumentos de defesa e planejarem o que e como irão apresentá-los. Além disso, também supõem os contra-argumentos para poderem levantar sua defesa. Antes do debate em si, estabelecem-se as regras quanto ao papel do moderador, forma de pedido de turno, tempo de turno para cada emissor, tolerância das interrupções, etc. Para finalizar, Ur lista possibilidades de temas para debate.

Um último material, que é preparatório para o exame CAE, intitulado “Advanced Listening \& Speaking”, cujo autor é K Gude apesar de não apresentar uma atividade específica de debate, propõe a prática de algumas funções (dar opinião, expor outro ponto de vista, perguntar se as pessoas 
concordam, etc.) ao longo das unidades para que os alunos possam progredir em seu desempenho na expressão oral (ver apêndice 5). Em uma das partes do exame oral, os alunos discutem um tema e devem chegar a um consenso.

Com base no quadro 1 , analisamos essas atividades encontradas em materiais para o de inglês como língua estrangeira para avaliarmos a adequação das propostas. Quanto ao plano de ação de linguagem, segundo o modelo didático consultado, reconhecemos a necessidade de se explorar o contexto de produção para que os agentes possam construir sentido em suas intervenções orais. Entretanto, não há atividades preparatórias para os alunos identificarem as características da situação de comunicação que envolve locutores e interlocutores em um debate.

No plano discursivo, os materiais se limitam a citar a necessidade de argumentos e contra-argumentos para a participação em um debate. Já as capacidades lingüístico-discursivas que compreendem, por exemplo, os mecanismos (reformulação, refutação) e as formulações (modalização, conectivos) não são trabalhadas. Em outras palavras, conforme vimos pelo modelo didático, os elementos selecionados para o ensino e que deveriam ser trabalhados nas aulas não aparecem como objetos de ensino nos materiais analisados para o ensino de inglês.

\section{c. Material para ensino da Língua Espanhola (apêndice 6)}

Durão e Oliveira propõem uma atividade sobre o gênero "Debate" no final do livro Español. Curso de Españolpara Hablantes de Português. Avanzado 1 (2001) (ver apêndice 6). A proposta apresenta, em primeiro lugar, uma definição de debate e uma explicação de sua organização - fase de preparação, fase de desenvolvimento e fase de conclusão. Além disso, há uma exposição de vocábulos tipicamente usados para expressar opinião, para contrapor a uma opinião e para moderar o debate (todas funções típicas de um debate). Em segundo lugar, propõe quatro atividades que 
envolvem a prática de debate. A primeira sugere que os alunos organizem a atividade sobre um tema atual. $\mathrm{Na}$ seqüência, a partir de um tema proposto, os alunos devem redigir frases que possam compor a apresentação do moderador sobre o tema. A questão seguinte, sugere que se listem palavras usadas pelo moderador para que a participação dos membros do debate seja solicitada. Finalmente, os alunos são direcionados a se dividir em grupos de 8 , sendo 3 membros a favor, 3 contra, 1 moderador e um secretário, para debaterem sobre um tema atual que escolherem.

Como podemos observar, há uma preocupação com a instrumentação dos alunos para a situação de comunicação em foco, já que as autoras fornecem explicações (sobre as fases), alguns exemplos (de frases introdutórios de argumentos, contra-argumentos e moderação) e exercícios preparatórios para a atividade (escolha de um tema, escrita de frases de apresentação e lista de palavras relacionadas ao tema). Tais práticas parecem estar voltadas para a preparação do debate que encerra essa seção. Dependendo da forma como o professor trabalhe, haverá uma prescrição de como agir em um debate, uma indicação das lexias apropriadas e uma pequena prática. Aparentemente, há intenção de experiência real com o gênero. Nesse caso, é importante que o trabalho seja tratado como uma construção e que o foco não esteja voltado somente para o produto final. Também seria importante fornecer informações para os alunos construírem seus argumentos e contra-argumentos por meio de leituras sobre o tema selecionado. Igualmente, os alunos poderiam gravar seus debates para que ao ouvi-los, pudessem fazer suas avaliações e críticas para poderem melhorar seu desempenho.

\section{Conclusão}

Alguns livros trazem atividades de discussão com o intuito de praticar a função argumentativa de dar e justificar sua opinião, concordar e discordar. 
Apesar de as propostas de alguns materiais visitados neste trabalho abordarem elementos que poderiam contribuir para a produção oral de um debate, o trabalho como um todo parece estar mais voltado para uma prática oral do que para a prática de um debate, de modo geral, excetuandose o material elaborado por Ur, dirigido ao ensino da língua inglesa e o de Durão e Oliveira voltado para o ensino do espanhol, nos quais há uma proposta mais especificamente ajustada à prática do debate.

Acreditamos que, em todos os casos vistos, a falta de um modelo didático no qual os autores pudessem se basear, assim como a não delimitação dos elementos centrais do gênero "Debate" a serem ensinados faz da maior parte dos materiais que abordam a temática uma compilação de atividades orais e não uma seqüência didática voltada para a aprendizagem das capacidades de linguagem mobilizadas para a aprendizagem do gênero em questão no presente trabalho.

\section{Referências Bibliográficas}

CITELLI, A. O texto argumentativo. São Paulo: Scipione, 1995. p. 7.

CRISTOVÃO, Vera Lúcia Lopes. Gêneros e ensino de leitura em LE: os modelos didáticos de gêneros na construção e avaliação de material didático. 2001. Tese (Doutorado em Lingüística Aplicada e Estudos da Linguagem). - Pontifícia Universidade Católica de São Paulo, São Paulo.

BAKHTIN, M. Marxismo e filosofia da linguagem. [Trad. M. Lahud e Y. F. Vieira] São Paulo: Hucietc, 1929, 1979.

BAKHTIN, M. Os gêneros do discurso. In: Estética da criação verbal. São Paulo: Martins Fontes, 1992. p. 279-326. (Coleção Ensino Superior).

BRONCKART, Jean-Paul. Atividade de linguagem, textos e discursos. Por um interacionismo sócio-discursivo. São Paulo: Educ, 1999. 
DOLZ, J.; SCHNEUWLY, B. Pour un enseignement de l'oral. Initiation aus genres formels à l'école. Paris: ESF Editeur, 1998. p. 27-46.

FÄITA, D. A noção de gênero discursivo em Bakthin. In: BRAIT, B. Bakthin, dialogismo e construção do sentido. Campinas: Editora da Unicamp, 1977. p. 159-177.

NASCIMENTO, E. L. O discurso na sala de aula: ressonâncias dialógicas nos movimentos enunciativos. Semina. Londrina, n. 21, p. 81-87, 2000.

O par adjacente pergunta-resposta no discurso.

SCHNEUWLY, B. Genres et types de discours: considérations psycholingiques et ontegénétiques. COLLOQUE DE L'UNIVERTISÉ CHARLES-DE-GAULLE, 3. Neuchâtel, 1994. p. 155-173.

.; DOLZ, J. Os gêneros escolares. Das práticas de linguagem aos objetos de ensino. [Trad. Glaís Sales Cordeiro] Revista Brasileira de Educação, n. 11, p. 5-11, maio/jun./jul./ago. 1999.

\section{Livros analisados}

ALEXANDER, L. G. For and against. Hong Kong: Longman, 1968/75.

BARBIERI DURÃO, A. B. A.; OLIVEIRA, M. E. O. Español. Curso de Español para Hablantes de Portugués. Avanzado 1. Madrid: Arco/Libros, 2001.

GUDE, K. Advanced Listening and Speaking. CAE. Oxford: Oxford University Press, 1999.

LADOUSSE, G. P. Speaking Personally. Quizzes and questionnaires for fluency practice. Cambridge: Cambridge University, 1983/88.

UR, P. Discussions that Work: Task-centred fluency practice. Cambridge: Cambridge University Press, 1981/1995. p. 105-108. 


\section{APÊNDICE 1}

\section{VIVÊNCLA E CONSTRUÇĀO}

\section{DEBATE}

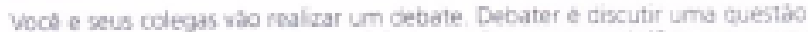

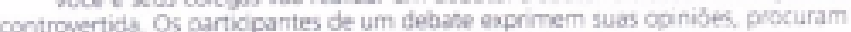
modficar as dos outros. nepensam suas posiçoes, para construa uma resposta comum a quesibo em drousso

Wea e analise as opirióes a respeito do tema Meninos e meninas devem te' aula de educacde fisca en turmas separados:

\section{Opinião 1}

Fu actso que a apeoweitamenoo das meminas e dos meninos on turmsas separadas é melhox. pois as exencicios poxdem sef sireckondodo Oberve que, no esponte em geral os times sako sempre femininos ou masculinose

\section{Opinião 2}

Nós vivemos em sociedade e cada vez mais estamsas fazendo tudo en mniumes. Homens, mulheres, criancas. peseras de idso mais avancadi participam de rudo o oue hă nas cidades. Devemos criar cada vez mais oportunidades paea todos aprenderem a fazer as atividades coletiomernte

\section{Opiniãa 3}

Fu acredito que 6 muito bom meninas e meninns estarem juntas duranke as atividades de educacto fisica, porque assim podem aprender uns com oh outros Por cutro labo, se exisem al guns momentos em que se noea que eles ou elas aprendem melhor separadamente, podem-se criar horlinios etin que estejam separados.

Forse, discuta com seus colegas e prolesser e escreva uma opinias a resperto desse assunto

2 No debate, ha vános papeis: apresentador, moderador, participantes e publico.

56 
Reconhec,a, nos trechos abaixo, esses papeis.

( Năo concordo com sua exposiça. Minha opiniào é exatamente a contrário disso.

conitratio

(6) O senhor tem um minuto para terminar seu pensamento e permitir as respostas dos outros participantes.

C Boa noite, senhoras e senhores. Estaremos aqui hoje debatendo sobre um axsunto polknico: Menores de 18 anos podem ou ndo dinigiv veicubs automotivos? Quviremos a opinido de alguns jovens, pais e especialistas

no assunto 9) Ha ainda alguém que gostaria de dizer algo a respeito desse ponto?

- Um carro nas máos de um menor de idade pode se transformar numa arma particasti

4 imagine que vock está participando de um debate sobre o uso ou nà de uniforme na escola. Escreva no caderno paragrafos que completem as sequintes expressßes: "Eu acho que"; "Ontem mesmo eu noter que"; "Eu compreendo seu ponto de vista, mas por outro lado", "Estou interamente de acordo com woce quando diz", "Vou citar um exemplo"; "N\$o concordo com vocé, pois".

4- Escreva um argumento a favor e um contra sobre a seguinte tema. Sav da sala de aula sem a necessidade da permissdo do professcr

bals de aute set

- Apora, reúna seu grupo e planejem um debate

- Escoham o terna.

- Pescusem sobre ele

- Escolham o apresentador o moderador e os participantes. O publico será a classe

- Orgariaen como serdo fetas as participaçces no debate

\section{Apresentador}

- cumprimento ao público e participantes.

- apresentaça do tema

- apresentacáo dos participantes.

- encerramento do debate e agradeximentos.

Participantes

- preparacso dos arqumentos da cpinilo contra ou a favce;

- preparacso para escutar e respeitar as autras opinióes, antes de contesta-las ou apciálas.

\section{Moderador}

- organizaça da ordem de apresentacaso das ideias,

- preparacajo para regulamentar o tempo de fala de cada participante,

- prepuracio e estudo scbre o tema, para comentar e encadear as opirices e os argumentos apresentados.

- Sentem-se numa mesa en frente a classe e realizem o debate.

SIGNUM: Estud. Ling., Londrina, n. 5, p. 125-157, dez. 2002 
APÊNDICE 2

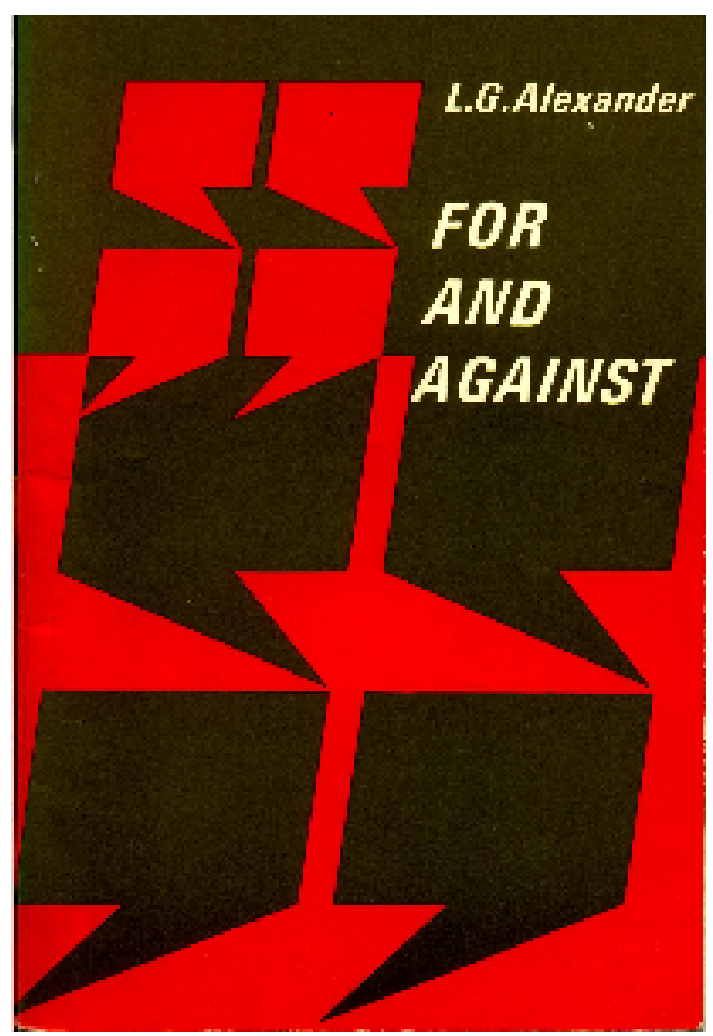




\section{I 'It's high time men ceased to regard women as}

second-class citizens'

This is supposed to be an enlightened age, but çou wouldn't think so if you could hear what the average man thinks of the average woman. Women won their independence years ago. After a long, bitter struggle, they now cnjoy the same educational opportunities as men in most parts of the

5 world. They hase prosed repearedly that they are equal and often superior to men in almost every ficld. The hard-fought bactle for recognition bas boen won, but it is by no means over. It is men, not women who still carry on the sex war bccausc their attitude remains basically hostile. Even in the most promressive socjetjes, women continue to be reganded as second-rate

Io citizens. To hear some men talk, you'd think that women belonged to a different species!

On the surface, the comments made by men sbout women's abilitics scem light-bearted. The same tired jokes abour women drivers are repeated day in, day out. This apperent light-heartedness does noc conocal

Is the real contempt that men feel for women. However much men sneer at somen, their elaims to superiority are not borne out by statistics. Let's consider the matter of driving, for instance. We all know that women cause far fewce accidents than men. They are too conscientious and responsible to driwe like maniacs. But this is a minor quibble. Women

20 bave succecded in any job you care to name. As policicians, soldiers, doctors, factory-hands, university professors, farmers, company directors, lawsers, bus-conductors, scientists and presidents of countrics they have often put men to shame. And we must remember that they frequently suoceced brilliantly in all these fields in addition to bearing and rearing

25 children.

Yet men go on maintaining the fiction that there are many jobs women can'r do. Top-level political negotiation between countries, busincss and banking are almost entirely controlled by men, who jealously guard their so-called 'rights". Even in otherwise enlightened places like Switzerland

30 women haven't even been given the vote. This situation is preposterous! The arguments that men put forward to exclude women from these fields are all too familiar. Women, they say, are unreliable and irracional, They depend too little on cool reasoning and mo much on intuition and instinct to arrive at decisions. They are not even capable of thinking

35 clearly. Yet when women prove their abilities, men refuse to acknowledge them and give them their duc. So much for a man's ability 0 think clearly!

The truth is that men cling to their supremacy beciuse of their basic inferiority complex. They shun real ompetition. They know in their

40 bearts that women are superior and they are afrajd of being beamen at their own game. One of the most important tasks in the world is to achieve peace between the nacions. You can be sure thac if women were allowed to sit round the conference cable, they would suoceed brilliantly, as they always do, where men have failed for centuries. Some things are too 45 important to be left to men! 


\section{The argument: key words}

I Supposed to be enlightened age: not really so.

2 Wounen won indepcndence years ago.

3 Long struggle: equal oducstional opportunities as men

4 Proved repestedly: equal, often superior to men in every field.

5 Batle not over: men carry on sex war; basically bostile.

6 Even in progressive sacieties: women second-rate cidzens; different specics!

7 Light-hesrted comments made by men: e.g. women drivers,

8 Does not conceil renl conoempt; but statistics disprose their claims.

9 Take driving: women; fewer accidents; responsiblc drivers, not manisca.

10 Success in any job: politicians, ecc, - bear and rear children as well.

II Men mainkain fiction: women can't do certain jobs.

I2 E.g.top-level political negotiation, banking, no vote in cctcain countries.

13 Why? Familiar argumencs: women unreliable, irrational, depend on instinct, intuition.

I4 Men refuse to acknowledge proven ability. Clenr thinking?

I5. Men cling to supremncy: inferiority complex.

I6 Shun oxmpetition; may be benten.

I7 Most important task; world peace.

18 Sucoess if negotiutions by women; some things too important to be done by men.

\section{The counter-argument: key words}

I Women: militunt, ahout touder because they have weak carse,

[ 2 Even now, thes still talk like suffragettes.

3 It's nunserse to claim that tnen nnd women ure equal and have the same nbilitics.

4 Women: different biological function; physically weaker; different, not inferior, intellectually.

5 Impossible to be wives, mothers and successful career tromen.

6 Really arz unreliable: employers can't trust them. Not their fault: leuve jobs to get married, have children.

[ 7 Great deal of truth in light-hearted jokes: e.g. women drivers. Women: less practical, less mechanjeally-dninded.

8 Most women glad to let men look nfter important aftairs.

9 They know that bearing and rearing children are more important,

to Thar's why there are few women in politics, etc. They are not excluded; they cxclude themselves.

II Anyway, we live in woman-doninated societies: e.g. USA, Western liurope.

I3 Who is the real boss in the aserape bousehold? Cerrainly not father!

I3 Nien are seoond-class citizens and women should grant them equal status! 
APÊNDICE 3

\section{Speaking Personally}

Qmizzes and questomiabs for fluesty practice

Gillian Porter Ladoussc

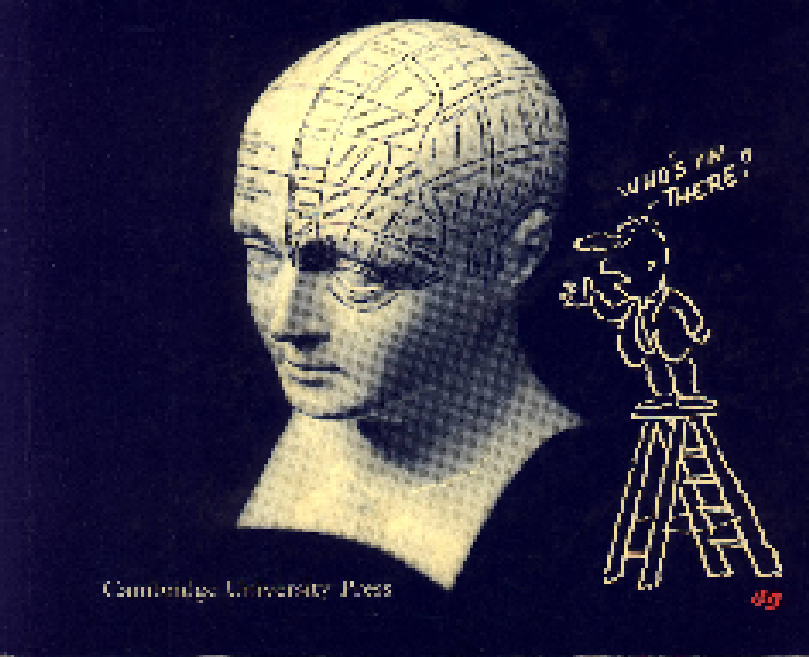

SIGNUM: Estud. Ling., Londrina, n. 5, p. 125-157, dez. 2002 


\subsection{Speaker's fimer}

As you walk past Hyde l’ark in I.ondon ros may see people speaking aut or subject that are dear to them. They are allowed to say what ther like aloout whom they like. Frecdom of specch is sacred. Pevple sometimes talk about important pulitical issues, such as unilateral disarmamene or the preservation of the enrironment, and sometimes abour more minor matess such as liversins hours in puts, the price of a dog licence, the legalisarion of sof drugs, cre.

In groups of six or seven, make your own Speaker's Cismer. Form a serui circle. l'lace a vacant shair in fronr of the group. Any member ot the gretrup is sllowed ro get up and sit in the emper chair and speak on the subject of his or her choice. A tirtse limit may be set.

\subsection{A debate}

Thehating is a popular activit' in British schools and universities. It is a tormal exchange of vicws on a subject, and an atcempt on the part of rhe speaker tos esnvert other people to his or her own poine sf vicw. $A$ dehate is carried out in rhe following way:

A motion is sel, for example; "This house helicves that censorship in the arts can newer be justified".

Two people volunter to propose the mostion, and ewo people to oppose the motichn.

The speakers cach prepare a twe-minute spech. They speak alccmately,

besinning with the proposer. The orher speakers may uf course have to modify what they say according to what the previous speakces have said.

"The house", or the public, is allowed txs ask questisms and finally a vote is taken to see whs has argued more convincingly.

Carry out a debace on one of rhe following subjects:

The treedom of the press should be absolute.

Easy divorec is a threat ro the stahility of suciety.

The death perally is a primitive rimal and shomld he abolished.

\subsection{Letter writing}

One way in which people express thcir social attitudes is by writing to newspapers. Look at these cxamples and write a similar letter on a subjecr about which you teel strongly.

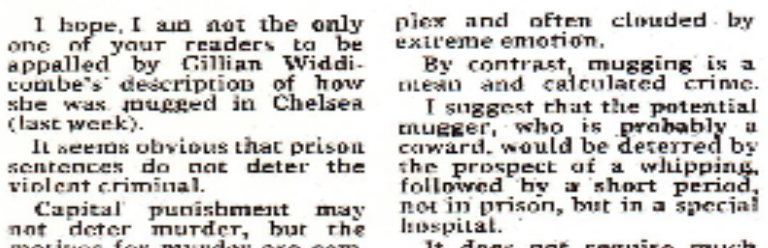
not detcr mutder, but the imazination to thlok of more

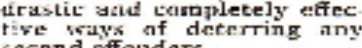
scennd nffenders.

The puaistument, nf vialence hs grenter piolence may seem brutal, but sutely it is ustived it tais is the onls wity to protect the physicalls weaker members of societ? The brataljty of thugs.

82 
Sit-We spend around E1,000 million a year on the results of family breakdown (Society Tomorrow,. December 17). The Government grant to the National Marriage Guidance Council is nowhere near fl million.

Fven a doubling of this would enable far more to be done: in wider research into those social and personal changes which help or hinder family life; an education programme with schools and other organisations to overcome the limentable lack of preparation noted by A. J. Brayshaw ; a substantial in crease in the number of counsellors

It is the children of broken or inadequate homes who go on to find the most difliculty in tmaking happy and lasting relationships. Do we want to see these patwe want to see these patduced until our supremacy in the divoree stakes

assured?

Donald Godder.

National Marriag

Guidance Council. Bristol.

Sir, - One would have thought that the cause of Hiss Lindi St. Claire, the London prostitute, is one our Women's Libbers would embrace. She herself makes it clear that she is not an exploited female. the object of men's lasts but an enterprising provider of humane prising provider of humane services to the weake members of the male sex. Yet what is virtually a socia service, and certainly the oldest known to civilisation is treated with that specifle hypoerisy for which the English are infamous.

How different from the West German code of conduct. Humburg's red light distriet, is regarded by the local authorities as a genuine place of business. The kindly plies who spend their windy ing hours there pay income 1.ax. and recejve the allowances which are their due. At the end of the day, they leave their "offices" and re. turn to apartments in respectable buildings in the residential areas of the city. Since prostitution is a to abolish, is it not more civilised to control it, than to close one's eyes to reality, and to pretend that it does not exist? This riew may be shocking opinion, but as we shocking opinton, but as we are part of the European understand ' why we should spurn the "mores" which the other members find acoeptable. - Yours faithfislly,

J. L. Hendeles.

Jo Cedars ciose.

Lontion N W4 
APÊNDICE 4

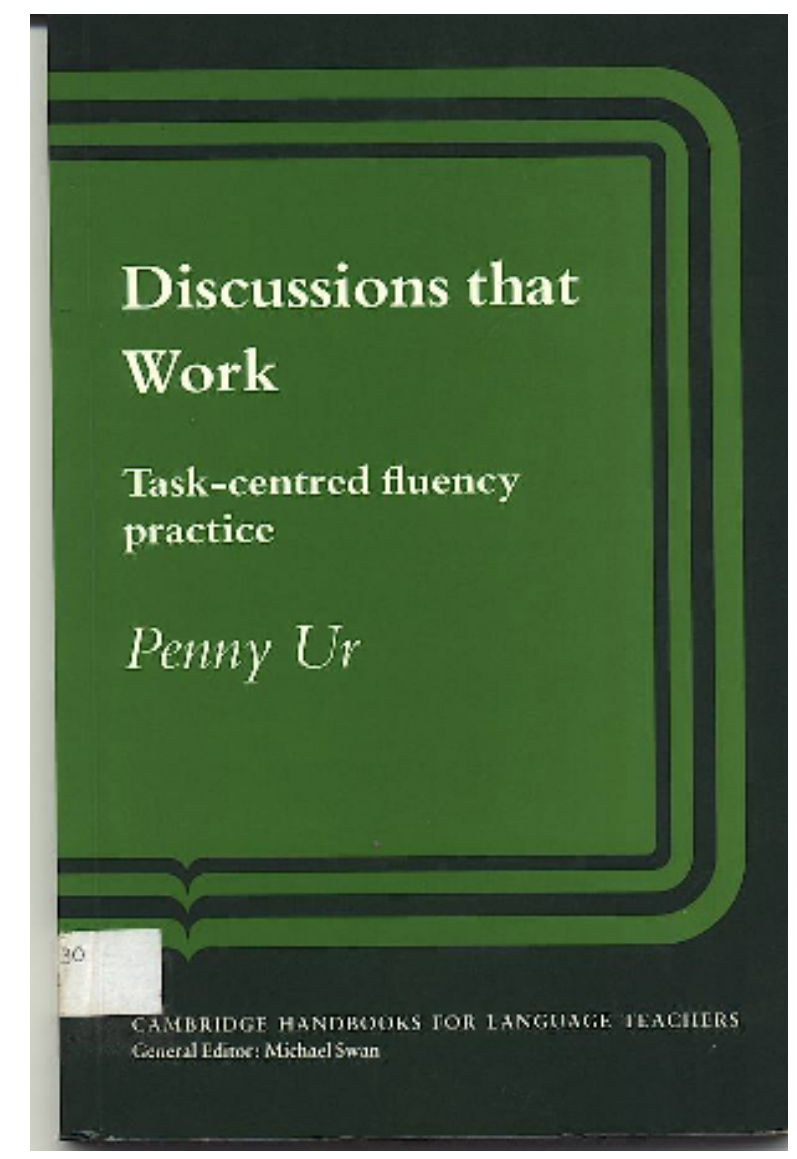




\section{Debates}

In Part 1, I discussed the limitations and disadvantages of the conventional class debate as a vehicle for fluency practice (pp. 5-6): the limited scope of its subjects (social, political or philosophical controversies), and the relative lack of participation (since it has to be a full-class, not a small-group activity). It would be a pity, however, to exclude the debate completely from our battery of communication exercises; many students (particularly the more adult and intellectual) enjoy this kind of discussion; and the skills of oratory and dialectics are learned and exercised in a debate better than anywhere else. Perhaps its disadvantages may be mitigated and its advantages preserved by using the following procedure.

The class is divided into two or three groups, each of whom is given a motion for debate: two of these are the direct opposite of each other (for example, 'Marriage is a perfect institution' and 'Marriage should be abolished'); and a third - optional - a compromise ('Marriage laws need reforming'). Alternatively, there may be four to six different motions, each one supporting a different point of view; then, of course, the class will be divided into the corresponding number of groups. In the 'balloon' debate, for example, four to six famous people are imagined to be hanging in a basket suspended from a balloon which is gradually deflating; one after another they will have to be thrown out to keep the balloon airborne, and ultimately only one will survive; 
the debate has to decide which person is most worth preserving. Other such sets of "competitive" subjects to be discussed and voted on are given in examples (n) to ( $r$ ).

In any case, whether there are two, three or several groups, the first stage in the activity is the same: each group has to work out and note down all possible arguments in favour of its motion (or candidate, in the 'balloon' debate), including defences against points that might be brought up by the opposition. It also has to work out the presentation of this material (who will put which argument and how), using every member of the group.

The groups can be divided according to the actual opinions of the students; but I have usually found it more effective to divide them arbitrarily, so that many will find themselves having to argue in favour of something they oppose, or vice-versa.

This is in fact a kind of role-play, and works very well as a rule; it also has the positive side-effect of making participants consider seriously the justifications behind other people's points of view. However. where students are deeply commicted or feel very strongly about some issue, they may resent being asked to argue strongly about some issue, they may resent to soing asked to so will probably be aware which subjects are likely to provoke such a reaction. In any case, it is not a good idea to choose a topic which excites violent opposition or defence; such excitement makes for disorganized debating and excessive use of the native language.

A time-limit should be set for the groups to prepare their cases - ten to fifteen minutes is usually enough - then students rearrange their seating to suit a full class discussion: in a circle, for example, or even in the conventional rows.

The degree of formality of the debate depends on the teacher and on what her class is used to: she may wish to adopt the eraditions of formal "parliamentary" procedure, or she may prefer a looser, less rigid structure, with only a chairman to regulate participation. If a proposer and seconder are going to be needed. then of course students should have been told this before their group preparation: other formalities of procedure can be outined by the teacher before the full debate begins. These should include such points as: what the chairman does, how participants indicate they want to speak, how long they may do so, how far interruptions are to be tolerated, and so on.

The full debate then ensues. It is to be hoped that all will participate, since each student will have been allotred a part of the "case" to put across, and the preparatory discussions supply both, or all, sides with plenty of ready "ammunition" for their speeches. Where students were originally divided into groups arbitrarily (not according to their real opinions), the teacher must 
use her own discretion as to whether they have to continue to support their group's motion during the entire debate, or whether they may be allowed to express their own views; it depends how far the debate is seen on the one hand as a game-like activity, and on the other as.a serious argument. In either case, the final voting should be 'genuine' (otherwise there is little point in it!); the announcement of the results of the vote constitutes the end of the activity.

Some examples of motions are given below. Examples (a) to (i) are for-and-against types, in which the opposing motion is actually written out as a counter-proposal implying a contradiction of the first; I have found this offers more stimulation for discussion, but it is optional: groups can simply be asked to support or oppose one proposition. The compromise suggestion which appears as the third proposition in examples $(j)$ to $(\mathrm{m})$ is also optional. Examples (n) to (r) are for use in the multiplechoice type of debate, such as the 'balloon' debate described above.

a) Love your country. Patriotism is outdated.

b) Everyone should be equal. Equality is neither possible nor desirable.

c) Children are little savages, tamed by the environment. Children are basically innocent and good, spoiled only by their environment.

d) Prisons should be abolished. More prisons should be built.

e) Nature gives us the best things in life. Civilization saves us from the cruelty of nature.

f) Riches make for happiness. Money can't make you happy.

g) Religion is the opium of the masses. Religion is the greatest force for moral good.

h) With age comes wisdom. Older is not necessarily wiser.

i) We learn through our mistakes. We learn by doing things right.

j) Marriage is a perfect institution. Marriage should be abolished. Marriage laws need reforming. 


\section{Compound activities}

k) The possession of marijuana should be illegal. Marijuana should be completely legalized. There should be some control over drug traffic.

1) A woman's place is in the home.

A woman's place is no more in the home than a man's is. Feminists will have to compromise over the woman's role in the family.

$\mathrm{m})$ There is no excuse for using corporal punishment in schools. Spare the rod and spoil the child.

Corporal punishment should be used only as a last resort.

n) (for a 'balloon' debate) The person most worth preserving from death is: a politician/a sportsman/a writer/a television personality/a scientist. (The teacher will, of course, use named personalities here, suitable to the cultural background of the students.)

o) The best place to live is: a flat/a caravan/a castle/a cottage/a tent.

p) The best hobby to have is: stamp-collecting/hiking/theatregoing/carpentry.

q) The most worthwhile subject to study is: English/science/ history/literature/psychology.

r) The best profession to have is: policeman/explorer/ teacher/politician/nurse. 
APÊNDICE 5

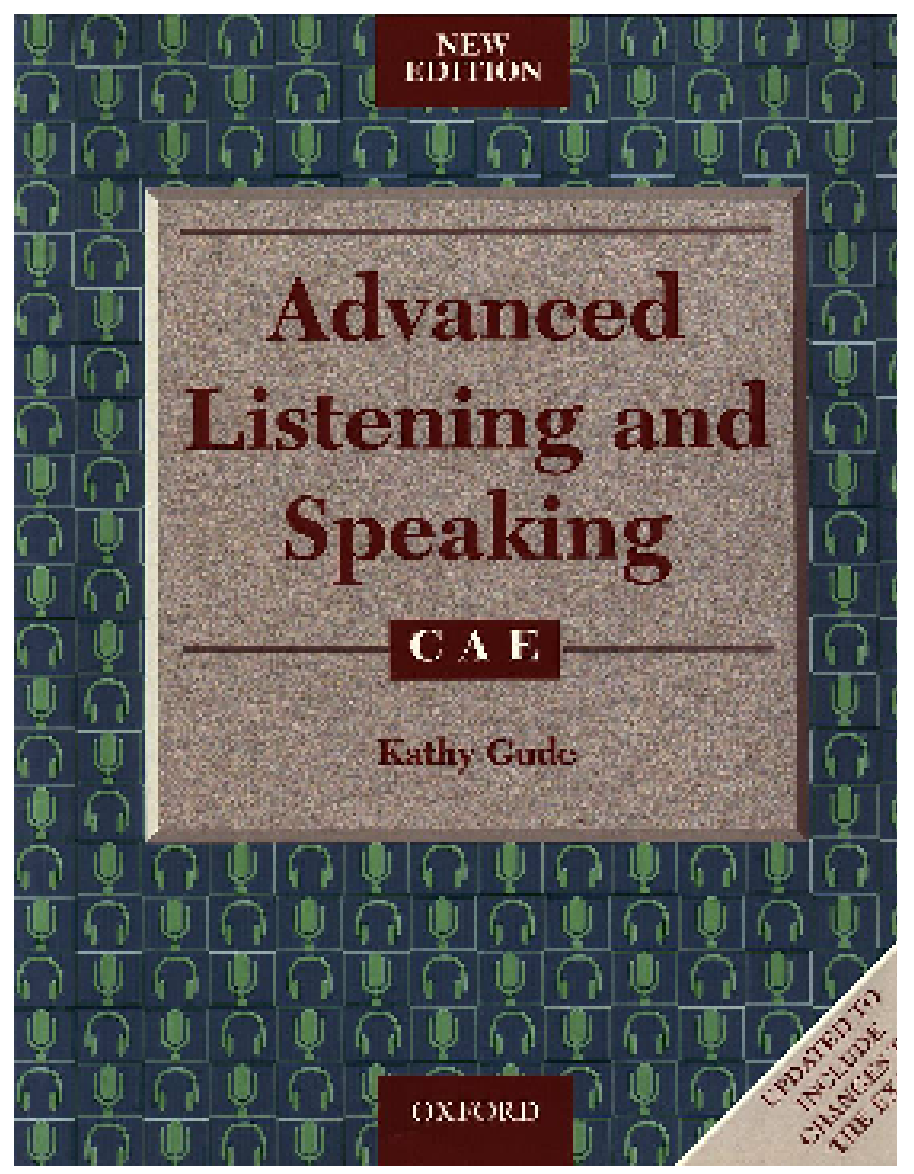

SIGNUM: Estud. Ling., Londrina, n. 5, p. 125-157, dez. 2002 
1C/D Jobs and training

Spotlight on Paper 5 Parts 3 and 4

(4) Exchanging opinions

Too young or too old?

Work through the Function File cards before doing the activity opposite.

\section{GIVING YOUR OPINION}

Which expression sounds rather rude and off-hand and suggests that you

have nothing else to say?

Which expresses a very strong opinion?

In my opinion / view ...

Generally speaking, I think...

Personally, I haven't the faintest idea about / whether...

To my mind. . .

Id just like to say. .

As far as I'm concerned...

I'm quite convinced that...

To be quite honest / frank...

If you ask me...

\section{ASKING FOR THE OPINION OF OTHERS}

Which expression is often used when addressing a group of people in a

more formal situation, e.g. a meeting?

Which expressions are often used when addressing an individual in a more

formal situation, e.g. a TV interview with a politician?

Which expressions are more suitable for an informal exchange of ideas?

What / How about you?

Would you care to comment on ... ?

Any comments?

Would you agree with that?

What are your views on...?

What's your opinion?

What do you think...? 
APÊNDICE 6

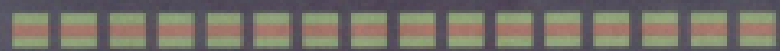

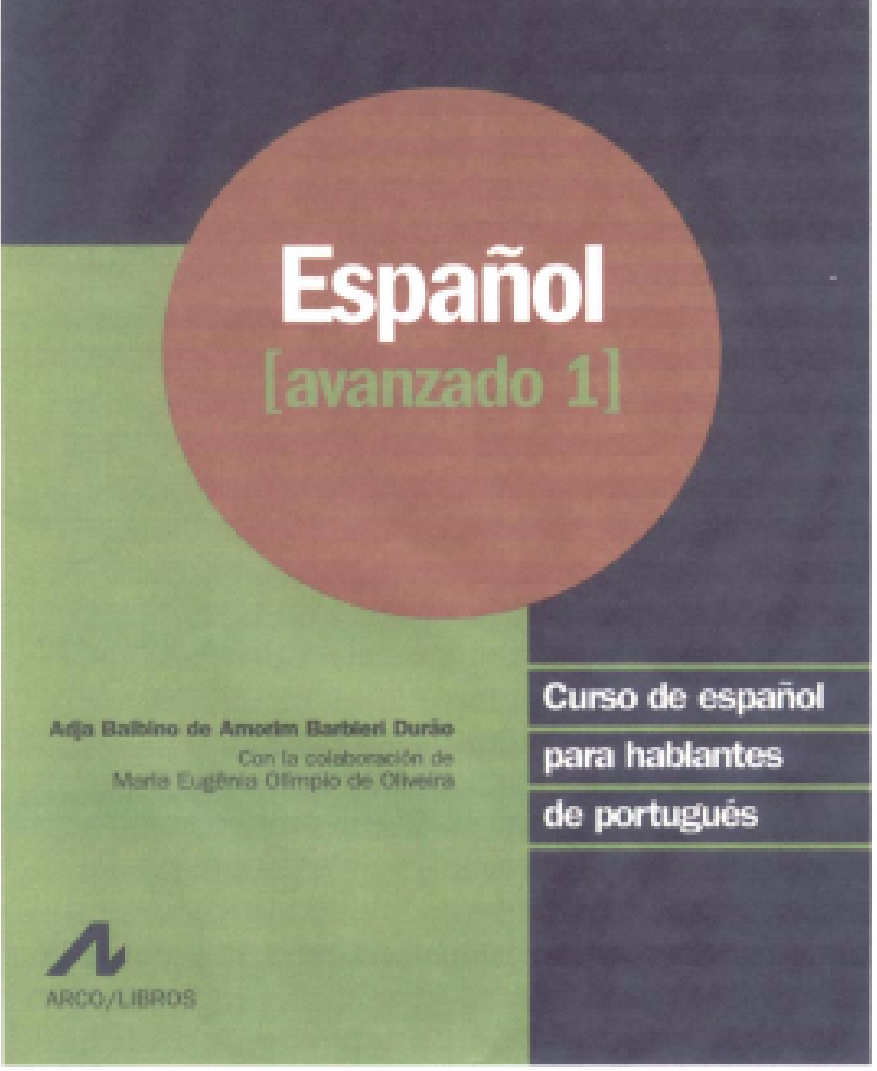

SIGNUM: Estud. Ling., Londrina, n. 5, p. 125-157, dez. 2002 


\section{Aprende
¡Dale a la lengua!
Organización de un debate
El debate es una conversación en la que las personas presentan diferentes puntos de vista so-
bre un tema previamente establecido con el fin de llegar a una puesta en común. Hay tres
fases en la organización de un debate. Apréndelas:}

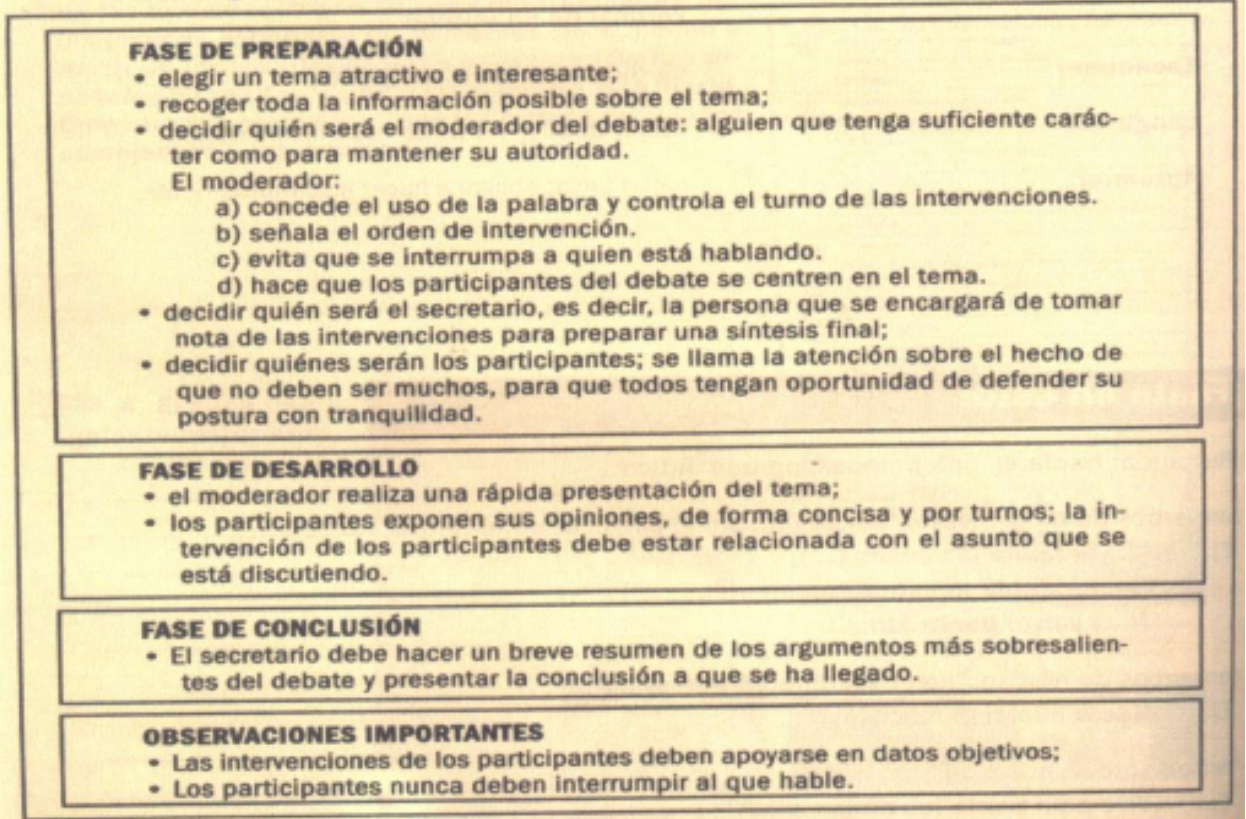

Para introducir: si se trata de expresar una opinión en el debate, se pueden emplear fórmulas como: "Para mí...", "En mi opinión...", "A mi modo de ver...", "Me parece que...", "Desde mi punto de vista...", etc.

Para contraponer una opinión a la formulada por otro participante se pueden utilizar: "Lamento no coincidir con la opinión de...". "Estoy en parte de acuerdo contigo, pero...", "Respecto a...", "Con relación a...", etc.

Moderador (para iniciar el debate): "Bueno, para empezar, vamos a discutir sobre...", "Vamos a hablar de...", etc. 


\section{Ahora, practica}

a 1 1. Organiza un debate sobre un tema actual.

Q 92 2. Jóvenes y adultos recurren al taco como medlo de expresín a dlarlo, hasta el punto de que se llegan a considerar como algo normal y corrlente. Cada vez más se emplean tacos y palabras malsonantes en buena parte de la programaclón. Redacta unas frases que sirvan para presentar como moderador el tema "Los tacos de la TV".

3. Redacta las palabras que emplearias como moderador para solicitar a un particlpante del debate que se ciña al tema en cuestín.

4. Participa con ocho companeros en un debate sobre un tema actual de vuestra elecclón, procedlendo del siguiente modo: Tres de vosotros formarb́ls el equipo A; otros tres, el equipo B; uno será el moderador y el último, el secretario. Uno de los equipos, según orlentación del moderador, expresará su punto de vista apoyándose en argumentos sólidos. El siguiente equipo, según orlentación del moderador, expresará su acuerdo o desacuerdo con lo que le ha dicho, aportando nuevos argumentos.

SIGNUM: Estud. Ling., Londrina, n. 5, p. 125-157, dez. 2002 